\title{
Stochastic vortex dynamics in two-dimensional easy-plane ferromagnets: Multiplicative versus additive noise
}

\author{
Till Kamppeter* and Franz G. Mertens ${ }^{\dagger}$ \\ Physikalisches Institut, Universität Bayreuth, D-95440 Bayreuth, Germany \\ Esteban Moro ${ }^{\ddagger}$ and Angel Sánchez ${ }^{\S}$ \\ Grupo Interdisciplinar de Sistemas Complicados (GISC), Departamento de Matemáticas, Universidad Carlos III de Madrid, \\ E-28911 Leganés, Madrid, Spain \\ A. R. Bishop** \\ Theoretical Division and Center for Nonlinear Studies, Los Alamos National Laboratory, Los Alamos, New Mexico 87545
}

(Received 24 September 1998)

\begin{abstract}
We study how thermal fluctuations affect the dynamics of vortices in the two-dimensional classical, ferromagnetic, anisotropic Heisenberg model depending on their additive or multiplicative character. Using a collective coordinate theory, we analytically show that multiplicative noise, arising from fluctuations in the local-field term of the Landau-Lifshitz equations, and Langevin-like additive noise both have the same effect on vortex dynamics (within a very plausible assumption consistent with the collective coordinate approach). This is a nontrivial result, as multiplicative and additive noises usually modify the dynamics quite differently. We also carry out numerical simulations of both versions of the model finding that they indeed give rise to very similar vortex dynamics. [S0163-1829(99)03917-X]
\end{abstract}

\section{INTRODUCTION}

In a large class of nonlinear problems arising in physics, chemistry, and biology, coherent, localized excitations often play a crucial role in governing the dynamics of the corresponding systems. This is the case, for instance, with solitons, vortices, fronts, and many other solitary wavelike objects found in a wide variety of low-dimensional systems. ${ }^{1-3}$ Physical situations featuring these phenomena are usually described by one of a few "canonical" partial differential equations, either integrable, such as the one-dimensional sine-Gordon or the nonlinear Schrödinger equations, or nonintegrable, such as the $\phi^{4}$ one. ${ }^{4}$ However, those mathematical formulations correspond in general to highly idealized physical contexts, in which factors like inhomogeneities, fluctuations, external fields, or damping are not taken into account. Conclusions about stability, dynamics, interactions, and any other property of coherent excitations drawn from those simple descriptions do not necessarily carry over when the above ingredients cannot be neglected. As a consequence, interest on the effect of perturbations on solitons and related excitations has grown rapidly since the early eighties, motivated by the need of bridging the gap between ideal models and real applications.

One of the most important and universal perturbations of any physical system is noise in one of its various forms. ${ }^{5}$ Typically, noise enters the physics of a system either as temporal fluctuations of internal degrees of freedom, caused by temperature, for instance, or as random variations of some external control parameter. In order to study the effect of these fluctuations in the system one is interested in, random terms are added to the original deterministic equations; generally speaking, internal randomness will reflect itself in $\mathrm{ad}$ ditive noise terms, while external fluctuations will almost always give rise to multiplicative noise terms. The difference between the two cases is that additive noise describes fluctuations independent of the values of the system variables, whereas multiplicative noise relates to fluctuations whose magnitude is modified by the state of the system. Of course, adding one kind of noise or the other to an otherwise deterministic problem leads in general to very different results, and nonlinear coherent excitations are not an exception: Thus, for instance, studies of the sine-Gordon ${ }^{6}$ and $\phi^{4}$ (Refs. 7,8 ) equations have shown that large scale (i.e., spatially homogeneous) noise modifies the dynamics of solitons in very different ways depending on its additive or multiplicative character. As another example, we note that the difference between additive and multiplicative noise in the nonlinear Schrödinger equation has also been discussed in Ref. 9, where multiplicative noise is associated with scattering of solitons by phonons with no creation of new phonons, whereas additive noise implies creation and annihilation of phonons. However, a general discussion of the analogies and differences of both kinds of fluctuations is lacking in nonlinear partial differential equations. Moreover, studies of noise effects on model systems are often carried out without any reference to the physical meaning of the type of term introduced; hence, we believe that a physically clear-cut example will help understand the similarities and differences of additive and multiplicative noise in other cases.

In this paper, we aim to gain insight into the effects of the two types of fluctuations by presenting a comparison of the effects of additive and multiplicative noise on the dynamics of vortices in two-dimensional (2D) easy-plane ferromagnets, as described by the classical, anisotropic Heisenberg model. The reason we choose this system is that we can justify physically in very direct ways the reasons for introducing one or the other type of noise in the Landau-Lifshitz 
equation, thus making possible the discussion and interpretation of our results in physical terms. To this end, we will address the problem both from analytical and numerical viewpoints in order to achieve a more complete picture of the two cases. Accordingly, in Sec. II we introduce our model, summarize its main features, and discuss how noise can be introduced in either form according to the physics one has in mind. In Sec. III, we present our analytical results, obtained in the framework of a collective coordinate approach. This approach will allow us to show the surprising result that, with a reasonable assumption, very natural in the context of a collective coordinate theory, the two kinds of fluctuations considered lead to the same dynamics for a single vortex. Our analytical predictions are tested in Sec. IV by means of numerical simulations, which yield a very good agreement with the theory, thus confirming a posteriori the validity of our assumption. Finally, Sec. V discusses our results and summarizes our main conclusions. It is important to note that, in principle, a formulation alternative to the one presented here is possible in terms of the Hamilton equations, instead of the Landau-Lifshitz equation. We discuss this possibility in the Appendix and show that it suffers from several problems.

\section{MODEL AND STOCHASTIC PERTURBATIONS}

The model we will be working with is the 2D anisotropic Heisenberg model with $X Y$ - or easy-plane symmetry, given by

$$
H=-J \sum_{\langle m, n\rangle}\left[S_{m}^{x} S_{n}^{x}+S_{m}^{y} S_{n}^{y}+(1-\delta) S_{m}^{z} S_{m}^{z}\right]
$$

where the subindices $x, y$, or $z$ stand for the spin components $0<\delta \leqslant 1$, and $\langle m, n\rangle$ labels nearest neighbors of a square lattice. Among its excitations, specially interesting ones are vortices, that are planar (i.e., with null $z$ component) if $\delta \geqslant 0.297$ and nonplanar (i.e., with localized $S_{z}$ structure) if $\delta \leqslant 0.297$. $^{10,11}$ Such nonplanar vortices will be the specific object of our study as reported in the remainder of the paper; however, the ideas we will be discussing are general enough to be of interest in other, related contexts where the system behavior is governed by solitonlike collective excitations.

Physically, this model has many interesting applications: In the last few years several classes of materials have been found or fabricated for which magnetic interactions within planes of their crystalline structure are much stronger than between these planes, and therefore the magnetic properties are basically 2D. Materials in these classes include, for instance, layered magnets (such as $\mathrm{Rb}_{2} \mathrm{CrCl}_{4}$ ), graphite intercalated compounds (such as $\mathrm{CoCl}_{2}$ ), magnetic lipid layers (such as manganese stearate), and high $T_{c}$ superconductors (see, e.g., Ref. 12). It is evident that in order to model better these materials, one of the factors that has to be added to a description in terms of the Heisenberg Hamiltonian is fluctuations, which can arise from different origins. Among those, thermal noise is of course the most natural source of fluctuations to consider in the context of the Heisenberg model: Indeed, from the experimental point of view, insofar as the motion of vortices has measurable consequences in inelastic neutron scattering ${ }^{13}$ and nuclear-magnetic reso- nance experiments, ${ }^{14}$ the effects of finite temperature on vortex dynamics can have signatures in those measurements.

In most cases, thermal effects are studied by adding to the equations of motion of the system under consideration an additive noise term plus a damping term in order to ensure that the fluctuation-dissipation theorem holds. This is simply the familiar Langevin dynamics. In our case, our starting (deterministic) point is the Landau-Lifshitz equation, which reads

$$
\frac{d \vec{S}_{m}}{d t}=-\vec{S}_{m} \times \frac{\partial H}{\partial \vec{S}_{m}}
$$

where $\vec{S}_{m}$ is the spin vector at lattice site $m$, and $H$ is the Hamiltonian, in our case that of the anisotropic Heisenberg model [Eq. (1)]. The corresponding Langevin dynamics equation for our model is obtained by adding damping and additive noise, which yields

$$
\frac{d \vec{S}_{m}}{d t}=-\vec{S}_{m} \times \frac{\partial H}{\partial \vec{S}_{m}}-\epsilon \vec{S}_{m} \times \frac{d \vec{S}_{m}}{d t}+\vec{\eta}_{m}(t) .
$$

The second term on the right-hand side of Eq. (3) is the damping term. Following Refs. 15-17, we have chosen for simplicity Gilbert damping, ${ }^{18}$ chiefly because it is isotropic in contrast to the Landau-Lifshitz damping. ${ }^{19}$ The last term in the right-hand side of Eq. (3) is the noise term, given by a Gaussian white noise with statistics defined by

$$
\begin{gathered}
\left\langle\eta_{m \alpha}(t)\right\rangle=0 \\
\left\langle\eta_{m \alpha}(t) \eta_{n \beta}\left(t^{\prime}\right)\right\rangle=D \delta_{m n} \delta_{\alpha \beta} \delta\left(t-t^{\prime}\right)
\end{gathered}
$$

where $D=2 \epsilon k_{\mathrm{B}} T$ is the diffusion constant and $\alpha, \beta=1,2,3$ denote Cartesian coordinates. It is important to note that, strictly speaking, the three Eqs. (3) do not represent Langevin equations, because all the components of $d \vec{S}_{m} / d t$ appear in each equation due to the cross products. To properly introduce the noise, one first has to group all the time derivatives on the left-hand side of the equation, and only then one can add independent white noise terms, say $\vec{\rho}_{m}$, for each spin component. In Refs. 20 and 21 it was shown that such a procedure leads to a different Langevin equation, in which the noise term $\vec{\eta}_{m}$ must be replaced by $\vec{\eta}_{m}=\vec{\rho}_{m}-\epsilon\left(\vec{S} \times \vec{\rho}_{m}\right)$. However, as discussed in Refs. 20 and 21, the correction is of the order of $\epsilon^{2}$, and taking into account that in the simulations values of $\epsilon$ of the order of $10^{-3}$ are used, the correction factor can be neglected, i.e., we take Eq. (3) as Langevin equation, containing purely additive noise.

What we have discussed is the usual way to introduce thermal fluctuations in any model. Nevertheless, in our case this Langevin approach suffers from the drawback that it enlarges the length of the spins, which in the original Heisenberg model is fixed to $|\vec{S}|=1$. In the simulations of Ref. 21, this unphysical effect was suppressed by renormalizing the length of every spin back to its original length at every time step. A more detailed discussion of the implementation of this constraint can be found in Ref. 20. At this point, we were not fully satisfied with this solution, and looked for another way to study thermal effects that preserve the spin 
length exactly. With this motivation, we now propose to introduce a noise term multiplicatively, according to

$$
\frac{d \vec{S}_{m}}{d t}=-\vec{S}_{m} \times\left[\frac{\partial H}{\partial \vec{S}_{m}}+\vec{h}_{m}(t)\right]-\epsilon \vec{S}_{m} \times \frac{d \vec{S}_{m}}{d t} .
$$

The term $\vec{h}_{m}(t)$ is again a set of independent Gaussian white noises, but now they represent fluctuations in the local field $\partial H / \partial \vec{S}_{m}$, in which the spin $\vec{S}_{m}$ precesses. In fact, this is a natural way to introduce the effect of thermal fluctuations, as the local field is the only way through which the spin $\vec{S}_{m}$ can feel any changes in its environment, due to those thermal fluctuations or for any other reason. Thus, the random term accounts for the interaction of the spin degrees of freedom with phonons, magnons, and any other excitation thermally generated. In addition, this fashion of introducing the noise has the property that Eq. (5) exactly preserves the spin length, hence there is no need for corrections as in the additive case. We note that a similar term has been considered by Garanin, $^{22}$ who proposed it in order to derive an alltemperature theory from the corresponding Fokker-Planck equation, obtaining a so-called Landau-Lifshitz-Bloch equation. Equation (5) has also been studied by García-Palacios and Lázcro, ${ }^{23}$ in connection with the dynamics of magnetic nanoparticles. Our purpose now is to use multiplicative noise in order to understand the influence of finite temperature on vortex dynamics, comparing the results with those arising from the usual Langevin approach discussed above.

There is an important question that deserves discussion before proceeding to the study of vortex dynamics, namely the correct way to interpret the stochastic partial differential Eqs. (3) and (5). The first one contains only additive noise, which implies that Ito or Stratonovic interpretations coincide, ${ }^{5,24}$ and therefore there is no problem in that case. As for the second one, being multiplicative, we do have to specify our interpretation of the equation. In principle, when thinking of thermal excitations interacting with the spins, we would have to associate with them a finite correlation time that would lead to a colored noise term. Taking white noise means taking the limit of zero correlation time, and therefore it is necessary to interpret Eq. (5) in the Stratonovic sense. Another reason for us to stay with this interpretation is that the spin modulus is conserved; it can be seen that Ito calculus leads to an exponential decrease of the modulus, with a damping time proportional to the damping $\epsilon$ in Eq. (5). In addition, in the Stratonovic interpretation, Garanin, ${ }^{22}$ and García-Palacios and Lázcro, ${ }^{23}$ and even earlier Brown, ${ }^{25}$ showed $^{26}$ that the stationary solution to the Fokker-Planck equation corresponding to Eq. (5) is the Boltzmann factor with Hamiltonian (1), indicating that Eq. (5) indeed represents the dynamics of our model at finite temperatures. Interestingly, it is not difficult to show that the Boltzmann factor is not a stationary solution to the Fokker-Planck equation for the additive noise Langevin problem [Eq. (3)], unless further assumptions are made, including the constraint of constant spin modulus. ${ }^{27}$

\section{ANALYTICAL RESULTS}

As stated in Sec. I, our approach to the problem of vortex dynamics will be both analytical and numerical. In this sec- tion, we first derive equations of motion for the vortex center $\vec{X}(t)$ both for the additive and for the multiplicative noise cases, and afterwards we compare with numerical simulations for our model, i.e., with results from numerical integration of Eqs. (3) and (5).

Our analytical approach to the stochastic dynamics of vortices begins by taking the continuum limit of Eqs. (3) and (5), which are much more difficult to deal with in a lattice formulation. This is a good approximation provided that the localized $S_{z}$ structure spans many lattice sites, as typically occurs in practice (except if the anisotropy parameter is chosen close to the critical value $\delta=0.297$ ). The next step is to use a collective coordinate theory to analyze the vortex dynamics (see Ref. 28 for a recent review on collective coordinate approaches). Within this procedure, one assumes that the shape of the excitation under consideration, in our case a vortex, is not modified by the perturbation for a large range of perturbation types (this is a very general, widely applicable $^{28}$ approach), i.e., in our case noise and damping terms, and that only the dynamics of its center is modified by these extra terms. The vortex motion is then introduced by the travelling wave Ansatz $\vec{S}(\vec{r}, t)=\vec{S}[\vec{r}-\vec{X}(t)]$, where $\vec{S}(\vec{r})$ describes the static vortex shape. Unfortunately, such a simple approach (first proposed for magnetic domains by Thiele ${ }^{15,16}$ ) is not enough to describe the vortex dynamics, as was found in Ref. 29. In that paper, Mertens et al. developed a generalization of the collective coordinate theory in which the vortex shape is allowed to depend on the velocity $\dot{\vec{X}}$ and, in general, also on higher-order derivatives of $\vec{X}(t)$. The corresponding generalized travelling wave Ansatz is

$$
\vec{S}(\vec{r}, t)=\vec{S}\left(\vec{r}-\vec{X}, \dot{\vec{X}}, \ddot{\vec{X}}, \ldots, \vec{X}^{(n)}\right),
$$

which yields an $(n+1)$ th order differential equation for $\vec{X}(t)$. However, as discussed in Ref. 29 , in the case of nonplanar vortices only the odd-order equations represent selfconsistent valid approximations; and it turns out that the third-order equation is sufficient to describe accurately all simulations without damping. ${ }^{29}$ Therefore, in this paper we use the Ansatz (6) with $n=2$ and apply it to the general case, which includes damping and noise.

The continuum versions of Eqs. (3) and (5) read, respectively

$$
\frac{d \vec{S}}{d t}=-\vec{S} \times \frac{\delta H}{\delta \vec{S}}-\epsilon \vec{S} \times \frac{d \vec{S}}{d t}+\vec{\eta}(\vec{r}, t)
$$

and

$$
\frac{d \vec{S}}{d t}=-\vec{S} \times\left[\frac{\delta H}{\delta \vec{S}}+\vec{h}(\vec{r}, t)\right]-\epsilon \vec{S} \times \frac{d \vec{S}}{d t} .
$$

To obtain the equations for the collective coordinate, instead of using the Hamiltonian procedure described in Ref. 29, we follow a much more direct approach, which we have already used for the additive noise (see a preliminary report in Refs. 20 and 30): We begin with Eq. (7) and multiply it by $\vec{S} \cdot\left(\partial \vec{S} / \partial X_{i}\right) \times$, where $X_{i}$ is the $i$ th component of the vortex center position. The contributions of all terms in the righthand side of Eq. (7) are 


$$
\begin{gathered}
-\vec{S} \cdot\left(\frac{\partial \vec{S}}{\partial X_{i}} \times\left[\vec{S} \times \frac{\delta H}{\delta \vec{S}}\right]\right)=-S^{2} \frac{\delta H}{\delta \vec{S}} \cdot \frac{\partial \vec{S}}{\partial X_{i}}=-S^{2} \frac{\partial \mathcal{H}}{\partial X_{i}}, \\
\epsilon \vec{S} \cdot\left[\frac{\partial \vec{S}}{\partial X_{i}} \times\left(\vec{S} \times \frac{d \vec{S}}{d t}\right)\right]=\epsilon S^{2} \frac{\partial \vec{S}}{\partial X_{i}} \cdot \frac{d \vec{S}}{d t}, \\
\vec{S} \cdot\left(\frac{\partial \vec{S}}{\partial X_{i}} \times \vec{\eta}\right)=\left(\vec{S} \times \frac{\partial \vec{S}}{\partial X_{i}}\right) \cdot \vec{\eta} .
\end{gathered}
$$

where $\mathcal{H}$ is the Hamiltonian density in the continuum limit of Eq. (1). According to our ansatz we insert in Eq. (9b)

$$
\frac{d \vec{S}}{d t}=\frac{\partial \vec{S}}{\partial X_{j}} \dot{X}_{j}+\frac{\partial \vec{S}}{\partial \dot{X}_{j}} \ddot{X}_{j}+\frac{\partial \vec{S}}{\partial \ddot{X}_{j}} \dddot{X}_{j} .
$$

The left-hand side of Eq. (7) is dealt with in the same way as just described for the right-hand side. By collecting the results, integrating over $\vec{r}$, and dividing by $S^{2}$, we obtain the same third-order equation as that in Refs. 20, 21, and 30:

$$
\begin{gathered}
(\mathbf{A}+\mathbf{a}) \dddot{\vec{X}}+(\mathbf{M}+\mathbf{m}) \ddot{\vec{X}}+(\mathbf{G}+\mathbf{g}) \dot{\vec{X}} \\
=\hat{\mathbf{A}} \ddot{\vec{X}}+\hat{\mathbf{M}} \ddot{\vec{X}}+\hat{\mathbf{G}} \dot{\vec{X}}=\vec{F}+\vec{F}^{\text {add }}
\end{gathered}
$$

The terms in Eq. (11) are as follows: The tensors denoted by capital letters come from the left-hand side of Eq. (7), and their expressions are, for the gyrotensor $\mathbf{G}$,

$$
G_{i j}=S^{-2} \int d^{2} r \vec{S} \cdot\left(\frac{\partial \vec{S}}{\partial X_{i}} \times \frac{\partial \vec{S}}{\partial X_{j}}\right),
$$

for the mass tensor $\mathbf{M}$,

$$
M_{i j}=S^{-2} \int d^{2} r \vec{S} \cdot\left(\frac{\partial \vec{S}}{\partial X_{i}} \times \frac{\partial \vec{S}}{\partial \dot{X}_{j}}\right),
$$

and for the third-order gyrotensor $\mathbf{A}$,

$$
A_{i j}=S^{-2} \int d^{2} r \vec{S} \cdot\left(\frac{\partial \vec{S}}{\partial X_{i}} \times \frac{\partial \vec{S}}{\partial \ddot{X}_{j}}\right) .
$$

The tensors denoted by small letters come from the Gilbert damping term; as can be seen from Eq. (11), they contribute to all orders, and they are given by

$$
\begin{aligned}
& g_{i j}=\epsilon \int d^{2} r \frac{\partial \vec{S}}{\partial X_{i}} \cdot \frac{\partial \vec{S}}{\partial X_{j}}, \\
& m_{i j}=\epsilon \int d^{2} r \frac{\partial \vec{S}}{\partial X_{i}} \cdot \frac{\partial \vec{S}}{\partial \dot{X}_{j}}, \\
& a_{i j}=\epsilon \int d^{2} r \frac{\partial \vec{S}}{\partial X_{i}} \cdot \frac{\partial \vec{S}}{\partial \ddot{X}_{j}}
\end{aligned}
$$

Finally, the force terms are

$$
F_{i}=-\int d^{2} r \frac{\partial \mathcal{H}}{\partial X_{i}}
$$

and

$F_{i}^{\mathrm{add}}=\frac{1}{S^{2}} \int d^{2} r\left(\vec{S} \times \frac{\partial \vec{S}}{\partial X_{i}}\right) \cdot \vec{\eta}(\vec{r}, t)$

The key to achieve a complete understanding of the vortex dynamics as described by Eq. (11) is to know the mean $\left\langle F_{i}^{\text {add }}\right\rangle$ and the variance $\operatorname{Var}\left(F_{i}^{\text {add }}\right)$. The mean is easily shown to be zero, whereas for the correlation functions, by using the continuum version of Eq. (4b) we obtain

$$
\left\langle F_{i}^{\mathrm{add}}(t) F_{j}^{\mathrm{add}}\left(t^{\prime}\right)\right\rangle=D \delta_{i j} \delta\left(t-t^{\prime}\right) \int d^{2} r \frac{\partial \vec{S}}{\partial X_{i}} \cdot \frac{\partial \vec{S}}{\partial X_{j}}
$$

We now turn to the multiplicative noise case Eq. (8). The calculations are mostly the same as described above, except that the contribution in Eq. (9c) is now substituted by the term coming from the multiplicative noise, which reads

$$
\vec{S} \cdot\left(\frac{\partial \vec{S}}{\partial X_{i}} \times(\vec{S} \times \vec{h})\right)=S^{2} \frac{\partial \vec{S}}{\partial X_{i}} \cdot \vec{h}
$$

As a consequence, only the stochastic term in Eq. (11) is modified: The new stochastic force is

$$
F_{i}^{\mathrm{mult}}=\frac{1}{S^{2}} \int d^{2} r \frac{\partial \vec{S}}{\partial X_{i}} \cdot \vec{h}(\vec{r}, t),
$$

which is in principle different from what we obtained for the additive case $F^{\text {add }}$. However, when we evaluate the first moments, we find again that the mean of $F^{\text {mult }}$ is zero. Furthermore, to compute the variance, we need to evaluate

$$
\begin{aligned}
& \left\langle F_{i}^{\text {mult }}(t) F_{j}^{\text {mult }}\left(t^{\prime}\right)\right\rangle \\
& \quad=\left\langle\int d^{2} r \int d^{2} r^{\prime} \frac{\partial S_{\alpha}}{\partial X_{i}} \frac{\partial S_{\beta}}{\partial X_{j}} h_{\alpha}(\vec{r}, t) h_{\beta}\left(\vec{r}^{\prime}, t^{\prime}\right)\right\rangle,
\end{aligned}
$$

where summation over $\alpha$ and $\beta$ is implied. At this point, it is important to notice that the noise is multiplicative, and therefore in principle we cannot take the spin fields out of the average over realizations of the noise. We stress that this is not a problem in the case of additive noise, and that we indeed proceeded that way to obtain the expression for the variance of $F^{\text {add }}$ given in Eq. (20). This is so because the additive character of the noise implies that the spin fields and the noise are uncorrelated. However, one cannot simply apply the same argument to the calculation in Eq. (23), because when the noise is multiplicative it is not clear whether the spin fields and the noise are correlated or not. In this situation, in principle we cannot exactly evaluate the variance of $F^{\text {mult }}$, but we can make the following approximation: At least for small noise, we can substitute the spin fields in Eq. (23) by the deterministic expression for the vortices, assuming reasonably that the corrections induced by the noise will be of the order of the noise strength and that their contribution to the variance would be two orders higher in the noise strength than that of the deterministic part, and hence, negligible. If we do so, we can then take the spin fields out of the average, finding 


$$
\left\langle F_{i}^{\mathrm{mult}}(t) F_{j}^{\mathrm{mult}}\left(t^{\prime}\right)\right\rangle=D \delta\left(t-t^{\prime}\right) \int d^{2} r \frac{\partial \vec{S}}{\partial X_{i}} \cdot \frac{\partial \vec{S}}{\partial X_{j}},
$$

i.e., the variance of the stochastic force due to the multiplicative noise is the same as that of the additive noise.

We stress that the assumption leading to the above, unexpected result, is very natural within a collective coordinate approach such as the one we are using here. To understand this, recall that the main hypothesis of a collective coordinate theory is that the shape of the excitation under study remains mostly unaffected by the perturbations, and only its position and possibly a few other collective variables change due to them. ${ }^{28}$ This assumption amounts to a drastic reduction in the degrees of freedom of the system: From an infinite number of them in the continuum equations to two for the center dynamics, in the present case. Physically, it is equivalent to neglect the contribution of the magnons ${ }^{31}$ excited by the perturbations to the vortex shape; and this approximation is made already at the very beginning, in order to obtain Eq. (11). In view of this, it would not be reasonable to keep the exact fields $\vec{S}$, containing the contribution of the phonon degrees of freedom, in Eq. (23), and therefore we carry out the calculation with the deterministic vortex shape, obtaining the result (24). ${ }^{32}$ Thus, consistently within our collective coordinate approach, we have shown that the mean and the variance of the stochastic forces induced by the multiplicative and the additive noises are the same (higher-order moments may still differ). As a consequence, the dynamics of a vortex is predicted to be the same under the influence of each type of noise, which is a surprising result in view of the general result that their effects are very different.

Once we have shown that both stochastic forces are equal, then Eq. (11) has to be the description of the vortex dynamics under the two types of noise. This equation was already studied and solved in Refs. 21 and 30, and we will only summarize here what we need for our present purposes. The classical spin is constrained to have a fixed magnitude that we set to unity. Therefore, the integrals on the right-hand side of Eqs. (12) through (17) were calculated using canonical fields $\phi=\arctan \left(S_{y} / S_{x}\right)$ and $\psi=S_{z}$ for the spin vector:

$$
\vec{S}=\sqrt{1-\psi^{2}} \cos \phi \vec{e}_{x}+\sqrt{1-\psi^{2}} \sin \phi \vec{e}_{y}+\psi \vec{e}_{z}
$$

Now, the explicit calculation of all the integrals is only possible if the dynamic structure of the vortex is known. In this respect, in Ref. 29 it was shown that the core region of the vortex contributes very little [except to Eq. (12)]; the dominant contributions stem from the outer region, if the system size is large enough. A vortex in the center of a circular system with radius $L$ and free-boundary conditions has the following structure in the outer region, which was confirmed by simulations: ${ }^{29}$

$$
\phi=\phi_{0}+\phi_{1}+\phi_{2}, \quad \psi=\psi_{0}+\psi_{1}+\psi_{2},
$$

with

$$
\begin{gathered}
\phi_{0}=q \tan ^{-1} \frac{x_{2}}{x_{1}}, \\
\phi_{1}=p\left(x_{1} \dot{X}_{1}+x_{2} \dot{X}_{2}\right),
\end{gathered}
$$

$$
\begin{gathered}
\phi_{2}=\frac{q}{8 \delta} \log \frac{r}{e L}\left(x_{2} \ddot{X}_{1}-x_{1} \ddot{X}_{2}\right), \\
\psi_{0} \sim p \sqrt{\frac{r_{v}}{r}} \exp \left(-r / r_{v}\right), \\
\psi_{1}=\frac{q}{4 \delta r^{2}}\left(x_{2} \dot{X}_{1}-x_{1} \dot{X}_{2}\right),
\end{gathered}
$$

and

$$
\psi_{2}=\frac{p}{4 \delta}\left(x_{1} \ddot{X}_{1}+x_{2} \ddot{X}_{2}\right)
$$

Here $q= \pm 1$ is the vorticity and $p= \pm 1$ is the polarization, which determines to which side the out-of-plane structure of the vortex points. In addition, $r_{v}=[(1-\delta) / \delta]^{1 / 2} / 2$ characterizes the radius of the vortex core. Straightforward integrations then yield expressions for the tensors $\hat{\mathbf{G}}, \hat{\mathbf{M}}$, and $\hat{\mathbf{A}}$, as well as for the forces, which can afterwards be inserted in Eq. (11) for the vortex motion. Finally, this equation is linear except for the force $\vec{F}(\vec{X})$, which can in turn be linearized by expanding around the mean trajectory. Subsequently, the equation can be solved by means of a Green's function approach and the so obtained solution can be used to calculate analytically the variances of the vortex trajectory $\mathbf{X}(t)$. These variances are proportional to the effective vortex diffusion constant

$$
D_{v} \simeq D \pi\left\{\log \frac{L}{a_{c}}+C\left(a_{c}\right)\right\},
$$

where $a_{c}$ is chosen of the order of $r_{v}$, and the unknown constant $C$ stems from the core region. As in this paper our main interest is to show that the dynamics under additive and multiplicative noises is the same, we will not dwell any further in the solution of Eq. (11); the interested reader is referred to Refs. 21 and 30 for details.

\section{NUMERICAL RESULTS}

Our numerical simulations begin with one vortex with its center located at a distance $R_{0}$ from the middle of a circularly shaped square lattice with a radius of $L$ lattice constants. We use free-boundary conditions to produce an image antivortex, which leads to a radial force on our vortex. ${ }^{29,33}$ The initial spin configuration is determined from an iterative program that produces a discrete vortex structure on the lattice. ${ }^{33}$ In this way we avoid the radiation of spin waves that would appear during the early time units if we use a continuum or other approximation for the vortex shape. The parameters we used in our simulations were: $\delta=0.1$, for the radius of the out-of-plane structure of the vortex to be large enough to avoid severe discreteness effects; system radius $L=24$ for the vortex to have enough space to evolve far from the boundaries; and initial distance from the circle center $R_{0}=10$.

For the time integration of the Landau-Lifshitz equation we use the discrete version of Eq. (3). Consistently with our interpretation of all the equations in the sense of Stratonovič, we use the Heun method to integrate in time. ${ }^{34,35}$ We explic- 

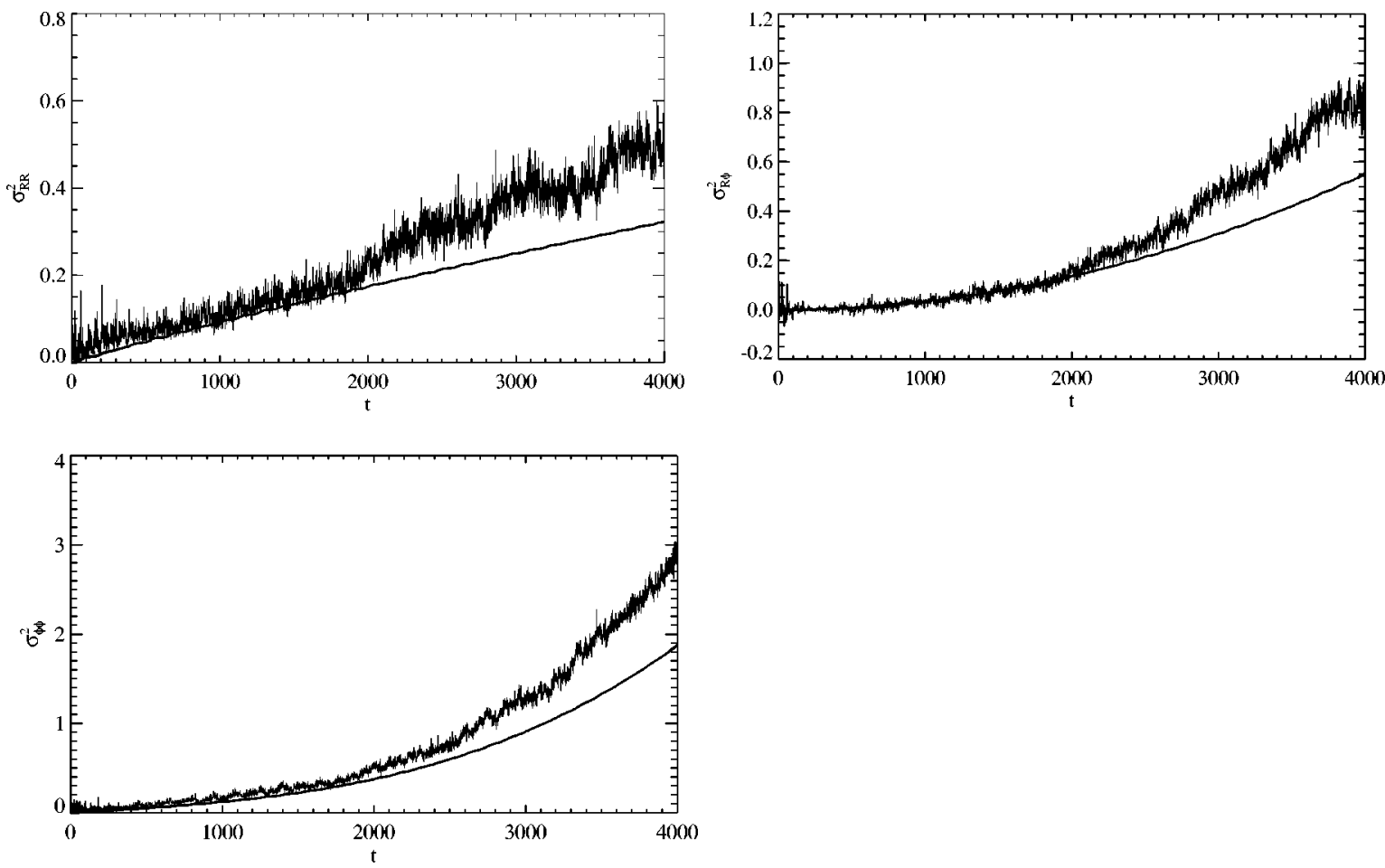

FIG. 1. Variances of the vortex trajectory for Langevin dynamics with additive noise. The temperature is $T=0.03$; other parameters are given in the text. From top to bottom, shown are the variance of the radial coordinate $\sigma_{R R}^{2}=\left\langle R^{2}\right\rangle-\langle R\rangle^{2}, \sigma_{R \phi}^{2}$, the off-diagonal elements of the variance matrix, and $\sigma_{\phi \phi}^{2}$, the variance of the azimuthal coordinate. In all three cases the lower line is the theoretical prediction with the vortex diffusion constant chosen in order to make the analytical curve lie just below the simulated data.

itly take into account the constraint $\vec{S}^{2}=1$ by means of a Lagrange parameter, as discussed in Ref. 36 (see also Ref. 21). To find a proper damping constant we checked the time dependence of the system energy using different damping constants for $L=24$ and $T=0.02$ (in dimensionless units). The energy at $t=0$ is the same as for $T=0$ and $\epsilon=0$ because the noise is introduced with the first time step of the simulation. The energy then rises and saturates to a value independent of $\epsilon$, but for $\epsilon>8 \times 10^{-3}$ the energy decreases slowly after saturation. The saturation time gets longer with lower $\epsilon$, for $\epsilon \geqslant 2 \times 10^{-3}$ we achieve acceptable saturation times $<300$ [in units of $\hbar /(J S)]$. We have always made a prerun of length $t_{0}>300$ prior to beginning the evaluation of the simulation data. Finally, our simulations consisted of numerical integrations up to times $t=4000$ (note in this regard that this takes ten days CPU time on a 433-MHz-DigitalAlpha workstation for averages over 100 runs) because this is larger than the characteristic time given by $5 / \epsilon$ for the damping in the trajectories. Finally, the difference between the energy without temperature and the saturation energy with temperature must be the thermal energy. We computed the mean thermal energy per spin at several temperatures and it agreed with $f / 2 \times k_{\mathrm{B}} T$ up to $T=0.9$ (for comparison, we note that the Kosterlitz-Thouless transition temperature is about 0.8 in our units), $f$ being the number of degrees of freedom per spin. This is far above the temperatures we will discuss below. We have to mention that for $T \gtrsim 0.1$ there is a more complex phenomenology because the thermal noise does not only induce a diffusive motion of the vortices but it can also flip their out-of-plane structure, correspondingly changing their direction of motion, and also nucleate additional vortex-antivortex pairs. In this paper we stay away from this regime, which has been considered in Ref. 37.

The outcome of our numerical simulations can be summarized by saying that they fully confirm the predictions of our analytical calculations, namely that the vortex dynamics is the same under both kinds of noise. An example of our results is shown in Fig. 1 for additive noise and in Fig. 2 for multiplicative noise. It is already evident from comparing the dispersion in the $R$ and $\phi$ components that the behavior of the vortex is very approximately equal in the two problems.

Furthermore, the comparison to the analytical prediction is very good: We emphasize that there is only one adjustable parameter, the vortex diffusion constant, whose value we can only estimate as an exact expression for the discrete structure of the vortex core is not known. The systematic deviation of the theory for very long times $(t \gtrsim 2000)$ is due to a simplification, ${ }^{21}$ namely keeping $R_{0}$ constant; in the simulation, $R_{0}$ slowly increases because of the damping. Results for other low temperatures compare equally well, the better the lower the temperature; for higher temperatures, our numerical estimates are less accurate, although qualitatively the results for both cases remain the same.

\section{CONCLUSIONS}

In this paper, we have provided analytical and numerical evidence that additive (Langevin-like) and multiplicative noise (coming from fluctuations in the local field) have the same effects on the dynamics of vortices described by the 2D Landau-Lifshitz-Gilbert equation. Analytically, the result has been obtained in the framework of a collective coordinate approach within a generalized travelling wave Ansatz. ${ }^{29}$ The variances of the effective force acting on the vortex were 

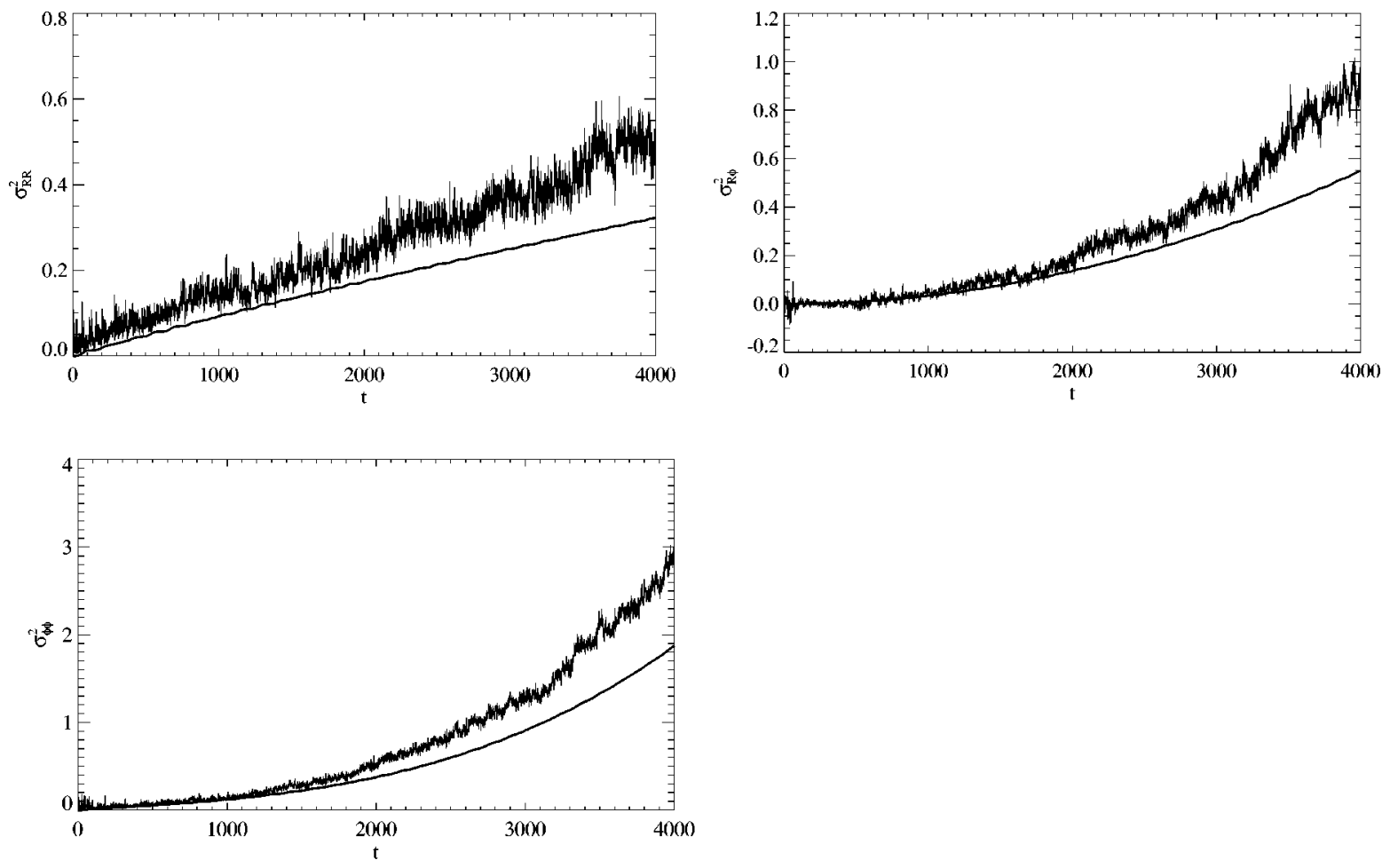

FIG. 2. Variances of the vortex trajectory for the case of multiplicative noise; parameters are the same as in Fig. 1. From top to bottom, shown are the variance of the radial coordinate, $\sigma_{R R}^{2}=\left\langle R^{2}\right\rangle-\langle R\rangle^{2}, \sigma_{R \phi}^{2}$, the off-diagonal elements of the variance matrix, and $\sigma_{\phi \phi}^{2}$, the variance of the azimuthal coordinate. In all three cases the lower line is the theoretical prediction with the vortex diffusion constant fitted to the data.

shown to be the same in the additive and multiplicative cases provided that deformations of the unperturbed vortex shape can be neglected. It is important to stress that this hypothesis is not an extra assumption but rather it is in fact implicitly made when using any collective coordinate approach. ${ }^{28}$ In order to substantiate our analytical results, we numerically simulated both the additive and the multiplicative cases for the 2D easy-plane Heisenberg ferromagnet, finding an excellent agreement with the prediction of equal behavior under both sources of noise, as well as with the analytical expression for the variance of the vortex trajectory.

As an immediate consequence of the validity of the above-discussed prediction, we point out that all the results obtained in Ref. 21 for the additive (thermal) noise apply to the multiplicative model presented here, specifically: (i) the existence of three different temperature regimes for the vortex propagation: a low temperature one, where the vortex motion follows essentially the third-order equation of motion with parameters independent of temperature; a middle temperature one, at which traces of the oscillations arising from the third-order equation are lost, and a high-temperature regime, which is not describable by a one-vortex approach because too many vortex-antivortex pairs arise in the system; and (ii) the dependence of the effective diffusion coefficient for the vortex on temperature. On the other hand, the experiments we have reported on here allow us to place on firmer ground that those are indeed the features of thermal vortex dynamics: The problem with the nonconstant spin length in a Langevin approach is now solved by the multiplicative approach, in which it is exactly conserved. Furthermore, the fact that the stationary probability distribution for the multiplicative case is precisely the Boltzmann factor reinforces our conclusion that this is the correct description of thermal effects in the framework of models with dynamics given by the Landau-Lifshitz-Gilbert equation. The thorough study carried out in Ref. 21 for the Langevin approach is then fully confirmed by the present paper.

\section{ACKNOWLEDGMENTS}

We thank Grant Lythe, Yuri Gaididei, Francisco Domínguez-Adame, Raúl Toral, and Niels Grønbech-Jensen for discussions. Travel between Bayreuth and Madrid was supported by "Acciones Integradas Hispano-Alemanas,' a joint program of DAAD (Az. 314-AI) and DGES. Additional funding for the stay of E.M. at Bayreuth was provided by the Fundación Universidad Carlos III. Travel between Europe and Los Alamos was supported by NATO Grant No. CRG 971090. Work at Leganés was supported by CICyT (Spain) Grant No. MAT95-0325 and by DGES (Spain) grant PB960119. Work at Los Alamos was supported by the United States Department of Energy.

\section{APPENDIX: NOISE IN THE HAMILTON EQUATIONS}

In this section we consider an alternative formulation of the problem of thermal fluctuations in the classical Heisenberg ferromagnet, and show that this version exhibits different features for additive and multiplicative noises, not even being well posed for the latter case.

By taking into account that the spin length has to be constant, one can reformulate the Heisenberg model in terms of the fields $\phi=\arctan \left(S_{y} / S_{x}\right)$ and $\psi=S_{z}$ for the spin vector. In this way, after rewriting the Hamiltonian (1) in these new 
variables, the dynamics of the model is governed by the following Hamilton equations:

$$
\begin{gathered}
\dot{\phi}=\frac{\delta H}{\delta \psi}, \\
\dot{\psi}=-\frac{\delta H}{\delta \phi} .
\end{gathered}
$$

The additive noise version of the problem, i.e., the Langevin equations, is given by

$$
\begin{gathered}
\dot{\phi}=\frac{\delta H}{\delta \psi}+\eta_{\phi}(\vec{r}, t), \\
\dot{\psi}=-\frac{\delta H}{\delta \phi}+\eta_{\psi}(\vec{r}, t),
\end{gathered}
$$

whereas the multiplicative version corresponds to

$$
\begin{gathered}
\dot{\phi}=\frac{\delta H}{\delta \psi}+\eta_{\phi}(\vec{r}, t) \phi, \\
\dot{\psi}=-\frac{\delta H}{\delta \phi}+\eta_{\psi}(\vec{r}, t) \psi .
\end{gathered}
$$

Of course, both models should also be supplemented with damping terms in order to fulfill the fluctuation-dissipation theorem. For simplicity, we will leave them out in this discussion. Their influence, however, is not qualitatively important as for our main argument below, as it can be checked that they would only contribute factors to the expressions for the force variances.

Now, from either one of these two equations we can derive an equation of motion for the vortex center following basically the same collective coordinate approach as above. In this case, one has to multiply the equation for $\dot{\psi}$ by $\partial \phi / \partial X_{i}$ and substract from it the equation for $\dot{\phi}$ times $\partial \psi / \partial X_{i}$. In this way an equation completely analogous to the third-order equation of motion (11) is obtained, but now we find for the forces in the additive case

$$
F_{i}^{\mathrm{add}}=\int d^{2} r\left[\frac{\partial \phi}{\partial X_{i}} \eta_{\psi}-\frac{\partial \psi}{\partial X_{i}} \eta_{\phi}\right],
$$

whereas for the multiplicative noise,

$$
F_{i}^{\mathrm{mult}}=\int d^{2} r\left[\frac{\partial \phi}{\partial X_{i}} \psi \eta_{\psi}-\frac{\partial \psi}{\partial X_{i}} \phi \eta_{\phi}\right] .
$$

Let us first discuss the additive case Eq. (A4). It is not difficult to derive from the above expression the first moments of the additive force, which turn out to be $\left\langle F_{i}^{\text {add }}(t)\right\rangle$ $=0$ and

$$
\left\langle F_{i}^{\mathrm{add}}(t) F_{i}^{\mathrm{add}}\left(t^{\prime}\right)\right\rangle=D \delta\left(t-t^{\prime}\right) \int d^{2} r\left[\left(\frac{\partial \phi}{\partial X_{i}}\right)^{2}+\left(\frac{\partial \psi}{\partial X_{i}}\right)^{2}\right]
$$

This expression is actually not very different from that obtained in Eq. (20) for the additive noise version of the Landau-Lifshitz equation: Using Eq. (25), the integrand of Eq. (20) reads, for $i=j$,

$$
\left(\frac{\partial \vec{S}}{\partial X_{i}}\right)^{2}=\left(1-\psi^{2}\right)\left(\frac{\partial \phi}{\partial X_{i}}\right)^{2}+\frac{1}{1-\psi^{2}}\left(\frac{\partial \psi}{\partial X_{i}}\right)^{2}
$$

As the static $S_{z}$ structure of the vortex (30) falls off exponentially in the outer region $a_{c} \leqslant r \leqslant L$, where $L$ is the system radius, both integrals (20) and (A6) have the same logarithmic size dependence,

$$
\left\langle F_{i}^{\mathrm{add}}(t) F_{i}^{\mathrm{add}}\left(t^{\prime}\right)\right\rangle=D \delta\left(t-t^{\prime}\right) \pi\left(\log \frac{L}{a_{c}}+C\right) .
$$

The only difference between both formulas consists in the value of the (small) constant $C$, which stems from the core region $0 \leqslant r \leqslant a_{c}$, where $a_{c}=\mathcal{O}\left(r_{v}\right)$.

Moving now to the multiplicative case, given by Eq. (A5), it can be easily shown that the situation is completely different. The mean and the variance of $F_{i}^{\text {mult }}$ can again be easily computed, yielding $\left\langle F_{i}^{\text {mult }}(t)\right\rangle=0$ and

$$
\begin{aligned}
\left\langle F_{i}^{\mathrm{mult}}(t) F_{i}^{\mathrm{mult}}\left(t^{\prime}\right)\right\rangle= & D \delta\left(t-t^{\prime}\right) \int d^{2} r\left[\left(\frac{\partial \phi}{\partial X_{i}}\right)^{2} \psi^{2}\right. \\
& \left.+\left(\frac{\partial \psi}{\partial X_{i}}\right)^{2} \phi^{2}\right]
\end{aligned}
$$

which, due to the presence of a factor $\psi^{2}$ in the first term of the integrand decaying exponentially away from the vortex core, is independent of the system size. It is then clear that the variances are very different in both cases. Therefore, a detailed study of the Hamilton equations with additive noise [Eqs. (A2)] would basically lead to the same conclusions summarized above arising from the study of the LandauLifshitz-Gilbert problem, whereas the multiplicative version would give totally different answers. It has to be concluded then that the proper way to include multiplicative noise in this problem is at the Landau-Lifshitz level and not in the Hamilton equations for $\phi$ and $\psi$. Finally, this result points out very clearly that the fact that the two approaches, additive and multiplicative, give the same results in the LandauLifshitz equation, is neither trivial nor generally applicable, and should then be regarded as a specific and attractive feature of that approach.

\footnotetext{
*Electronic address: till.kamppeter@theo.phy.uni-bayreuth.de †Electronic address: franz mertens@ @heo.phy.uni-bayreuth.de †Electronic address: emoro@math.uc3m.es

${ }^{\S}$ Electronic address: anxo@math.uc3m.es

**Electronic address: arb@viking.lanl.gov

${ }^{1}$ Nonlinearity in Condensed Matter, edited by A. R. Bishop, R.
}

Ecke, and J. Gubernatis (Springer, Berlin, 1993).

${ }^{2}$ Nonlinear Coherent Structures in Physics and Biology, edited by K. H. Spatschek and F. G. Mertens (Plenum, New York, 1994).

${ }^{3}$ Fluctuation Phenomena: Disorder and Nonlinearity, edited by A. R. Bishop, S. Jiménez, and L. Vázquez (World Scientific, Singapore, 1995). 
${ }^{4}$ M. Remoissenet, Waves Called Solitons (Springer, Berlin, 1995).

${ }^{5}$ N. van Kampen, Stochastic Processes in Physics and Chemistry (North-Holland, Amsterdam, 1980).

${ }^{6}$ P. J. Pascual and L. Vázquez, Phys. Rev. B 32, 8305 (1985).

${ }^{7}$ M. J. Rodríguez-Plaza and L. Vázquez, Phys. Rev. B 41, 11437 (1990).

${ }^{8}$ A. Sánchez, L. Vázquez, and V. V. Konotop, Phys. Rev. A 44, 1086 (1991); V. V. Konotop, A. Sánchez, and L. Vázquez, Phys. Rev. B 44, 2554 (1991).

${ }^{9}$ O. Bang, P. L. Christiansen, F. If, and K. Ф. Rasmussen, Applicable Analysis 57, 3 (1995); see also O. Bang, P. L. Christiansen, F. If, K. Ф. Rasmussen, and Y. B. Gaididei, Phys. Rev. E 49, 4627 (1994).

${ }^{10}$ M. E. Gouvea, G. M. Wysin, A. R. Bishop, and F. G. Mertens, Phys. Rev. B 39, 11840 (1989).

${ }^{11}$ G. M. Wysin, Phys. Lett. A 240, 95 (1998).

${ }^{12}$ A. R. Völkel, F. G. Mertens, A. R. Bishop, and G. M. Wysin, Phys. Rev. B 43, 5992 (1991).

${ }^{13}$ K. Hirakawa, H. Yoshizawa, J. D. Axe, and G. Shirane, J. Phys. Soc. Jpn. 52, 19 (1983); L. P. Regnault, J. P. Boucher, J. RossatMignod, J. Bouillot, R. Pynn, J. Y. Henry, and J. P. Renard, Physica B \& C 136, 329 (1986); M. T. Hutchings, P. Day, E. Janke, and R. Pynn, J. Magn. Magn. Mater. 54-57, 673 (1986); S. T. Bramwell, M. T. Hutchings, J. Norman, R. Pynn, and P. Day, J. Phys. (Paris) Colloq. 49, C8-1435 (1988); D. G. Wiesler, H. Zabel, and S. M. Shapiro, Physica B 156-157, 292 (1989); D. G. Wiesler, H. Zabel, and S. M. Shapiro, Z. Phys. B 93, 277 (1994); L. P. Regnault, C. Lartigue, J. F. Legrand, B. Farago, J. Rossat-Mignod, and J. Y. Henry, Physica B 156-157, 298 (1989).

${ }^{14}$ P. Gaveau, J. P. Boucher, L. P. Regnault, and Y. Henry, J. Appl. Phys. 69, 6228 (1991).

${ }^{15}$ A. A. Thiele, Phys. Rev. Lett. 30, 230 (1973).

${ }^{16}$ A. A. Thiele, J. Appl. Phys. 45, 377 (1974).

${ }^{17}$ D. L. Huber, Phys. Rev. B 26, 3758 (1982).

${ }^{18}$ The sign of our damping term differs from Refs. 15, 16, and 19 because we work with spins while these authors deal with magnetizations.

${ }^{19}$ S. Iida, J. Phys. Chem. Solids 24, 625 (1963).

${ }^{20}$ T. Kamppeter, F. G. Mertens, A. Sánchez, N. Grønbech-Jensen, A. R. Bishop, and F. Domínguez-Adame, in Theory of Spin Lattices and Lattice Gauge Models, edited by J. W. Clark and M. L. Ristig, Lecture Notes in Physics Vol. 494 (Springer, Berlin, 1997), p. 147.

${ }^{21}$ T. Kamppeter, F. G. Mertens, A. Sánchez, A. R. Bishop, F. Domínguez-Adame, and N. Grønbech-Jensen, Eur. Phys. J. B 7, 607 (1999).
${ }^{22}$ D. A. Garanin, Phys. Rev. B 55, 3050 (1997).

${ }^{23}$ J. L. García-Palacios and F. J. Lázcro, Phys. Rev. B 58, 14937 (1998).

${ }^{24}$ C. W. Gardiner, Handbook of Stochastic Methods (Springer, Berlin, 1990).

${ }^{25}$ W. F. Brown, Jr., Phys. Rev. 130, 1677 (1963).

${ }^{26}$ In fact, this result is for the Landau-Lifshitz equation with Landau damping. However, one can reproduce the calculations in, e.g., Garanin's paper (Ref. 22) (although they become somewhat more involved) to check that the result holds for Gilbert damping as well.

${ }^{27}$ T. Kamppeter, Ph.D. thesis, University of Bayreuth, 1999.

${ }^{28}$ A. Sánchez and A. R. Bishop, SIAM Rev. 40, 579 (1998).

${ }^{29}$ F. G. Mertens, H.-J. Schnitzer, and A. R. Bishop, Phys. Rev. B 56, 2510 (1997).

${ }^{30}$ T. Kamppeter, F. G. Mertens, A. Sánchez, F. Domínguez-Adame, A. R. Bishop, and N. Gronbech-Jensen, in Proceedings of the VIII Spanish Meeting on Statistical Physics “FisEs '97,', edited by J. A. Cuesta and A. Sánchez (Editorial del CIEMAT, Madrid, 1998), p. 133.

${ }^{31}$ To be precise, not all the magnons are neglected. As discussed in B. A. Ivanov, H. J. Schnitzer, F. G. Mertens, and G. M. Wysin, Phys. Rev. B 58, 8464 (1998), the generalized traveling wave Ansatz (Ref. 29) can be shown to be equivalent to a description in terms of a rigid travelling wave plus a few quasilocal translational magnon modes. Thus, the Ansatz in Ref. 29 indeed incorporates some magnon modes but, of course, an infinite number of them is still neglected.

${ }^{32}$ It is important to have in mind that this approximation can be done for any kind of perturbation, at least in principle. Either stochastic or deterministic terms generally give rise to the appearance of linear excitations in the system, and those are the ones neglected by the collective coordinate theory. Therefore, this is not a particular property of our stochastic perturbative terms, and no use of the specific nature of the perturbation terms is needed to arrive at the conclusion.

${ }^{33}$ H.-J. Schnitzer, Ph.D. thesis, University of Bayreuth, 1996.

${ }^{34}$ T. C. Gard, Introduction to Stochastic Differential Equations (Marcel Dekker, New York, 1987), Vol. 114.

${ }^{35}$ M. San Miguel and R. Toral, in Nonequilibrium Structures VI, edited by E. Tirapegui (Kluwer Academic, Dordrecht, 1998).

${ }^{36}$ N. Gronbech-Jensen and S. Doniach, J. Comput. Chem. 15, 997 (1994).

${ }^{37}$ Y. Gaididei, T. Kamppeter, F. G. Mertens, and A. R. Bishop, Phys. Rev. B 59, 7010 (1999). 